\title{
MiR-26a-5p inhibits GSK3 $\beta$ expression and promotes cardiac hypertrophy in vitro
}

\author{
Liqun Tang ${ }^{\text {Corresp., } 1}{ }^{1}$,jianhong Xie ${ }^{1}$, Xiaoqin Yu ${ }^{2}$, Yangyang Zheng ${ }^{1}$ \\ ${ }^{1}$ Department of Geriatrics, Zhejiang Province People's Hospital, Hangzhou Medical College, Hangzhou, Zhejiang, China \\ 2 Department of Geriatrics, Zhejiang Aid Hospital, Hangzhou, Zhejiang, China \\ Corresponding Author: Liqun Tang \\ Email address: tangyz0594@163.com
}

Background: The role of miR-26a-5p expression in cardiac hypertrophy remains unclear. Herein, the effect of miR-26a-5p on cardiac hypertrophy was investigated using phenylephrine (PE)-induced cardiac hypertrophy in vitro and in a rat model of hypertension-induced hypertrophy in vivo.

Methods: The PE-induced cardiac hypertrophy models in vitro and vivo were established. To investigate the effect of miR-26a-5p activation on autophagy, the protein expression of autophagosome marker (LC3) and p62 was detected by western blot analysis. To explore the effect of miR-26a-5p activation on cardiac hypertrophy, the relative mRNA expression of cardiac hypertrophy related mark GSK3 $\beta$ was detected by qRT-PCR in vitro and vivo. In addition, immunofluorescence staining was used to detect cardiac hypertrophy related mark $\alpha$-actinin. The cell surface area was measured by immunofluorescence staining. The direct target relationship between miR-26a-5p and GSK3 $\beta$ was confirmed by dual luciferase report.

Results: MiR-26a-5p was highly expressed in PE-induced cardiac hypertrophy. MiR-26a-5p promoted LC3II and decreased p62 expression in PE-induced cardiac hypertrophy in the presence or absence of lysosomal inhibitor. Furthermore, miR-26a-5 $p$ significantly inhibited GSK3 $\beta$ expression in vitro and in vivo. Dual luciferase report results confirmed that miR-26a-5p could directly target GSK3 $\beta$. GSK3 $\beta$ overexpression significantly reversed the expression of cardiac hypertrophy-related markers including ANP, ACTA1 and MYH7. Immunofluorescence staining results demonstrated that miR-26a-5p promoted cardiac hypertrophy related protein $\alpha$-actinin expression, and increased cell surface area in vitro and in vivo.

Conclusion: Our study revealed that miR-26a-5p promotes myocardial cell autophagy activation and cardiac hypertrophy by regulating GSK3 $\beta$, which needs further research. 
$1 \quad$ MiR-26a-5p inhibits GSK3ß expression and promotes cardiac hypertrophy in

2

3 Running title: MiR-26a-5p promotes cardiac hypertrophy

4 Authors:

5 Liqun Tang ${ }^{*}, 1$ : tangyz0594@163.com

6 Jianhong Xie ${ }^{1}$ : jhongxie@163.com

7 Xiaoqin $\mathrm{Yu}^{2}$ : yxq4027205@163.com

8 Yangyang Zheng1: fanniezhengyy@163.com

9 1. Department of Geriatrics, Zhejiang Province People's Hospital, Hangzhou Medical College, 10 No.156 Shangtang Road, Xiacheng District, Hangzhou 310014, Zhejiang, P R China.

2. Department of Geriatrics, Zhejiang Aid Hospital, No.509 Dongxin Road, Xiacheng District, Hangzhou 310016, Zhejiang, P R China.

\section{* Corresponding author: Liqun Tang}

E-mail: tangyz0594@163.com

Department of Geriatrics, Zhejiang Province People's Hospital, Hangzhou Medical College, No.156 Shangtang Road, Xiacheng District, Hangzhou 310014, Zhejiang, P R China.

\section{Abstract:}

Background: The role of miR-26a-5p expression in cardiac hypertrophy remains unclear. Herein, the effect of miR-26a-5p on cardiac hypertrophy was investigated using phenylephrine (PE)-induced cardiac hypertrophy in vitro and in a rat model of hypertension-induced hypertrophy in vivo.

Methods: The PE-induced cardiac hypertrophy models in vitro and vivo were established. To investigate the effect of miR-26a-5p activation on autophagy, the protein expression of autophagosome marker (LC3) and p62 was detected by western blot analysis. To explore the effect of miR-26a-5p activation on cardiac hypertrophy, the relative mRNA expression of cardiac hypertrophy related mark GSK $3 \beta$ was detected by qRT-PCR in vitro and vivo. In addition, immunofluorescence staining was used to detect cardiac hypertrophy related mark $\alpha$ actinin. The cell surface area was measured by immunofluorescence staining. The direct target relationship between miR-26a-5p and GSK3 $\beta$ was confirmed by dual luciferase report.

Results: MiR-26a-5p was highly expressed in PE-induced cardiac hypertrophy. MiR-26a-5p promoted LC3II and decreased p62 expression in PE-induced cardiac hypertrophy in the presence or absence of lysosomal inhibitor. Furthermore, miR-26a-5p significantly inhibited GSK3 $\beta$ expression in vitro and in vivo. Dual luciferase report results confirmed that miR-26a-5p could directly target GSK $3 \beta$. GSK $3 \beta$ overexpression significantly reversed the expression of cardiac hypertrophy-related markers including ANP, ACTA1 and MYH7. Immunofluorescence 
staining results demonstrated that miR-26a-5p promoted cardiac hypertrophy related protein $\alpha$ actinin expression, and increased cell surface area in vitro and in vivo.

Conclusion: Our study revealed that miR-26a-5p promotes myocardial cell autophagy activation and cardiac hypertrophy by regulating GSK $3 \beta$, which needs further research.

Key words: MiR-26a-5p; cardiac hypertrophy; autophagy; GSK3 $\beta$; LC3; $\alpha$-actinin

\section{Introduction:}

Cardiac hypertrophy is a major risk factor for cardiovascular morbidity and mortality, characterized by increasing heart mass, protein synthesis, and re-expression of fetal-type genes. Cardiac overload and injury often contribute to cardiac hypertrophy. Though initially compensatory, long-term cardiac hypertrophy often induces heart failure that is a common cause of death in the world (Wang et al. 2012; Zhang et al. 2016a). Its pathological process is closely related to the complex changes of cardiac gene expression patterns and changes in molecular pathways (Tang et al. 2017). However, the regulation mechanism remains unclear (Yang et al. 2015).

As an evolutionarily conserved process, autophagy plays a key role in maintaining cardiac homeostasis. It has been confirmed that autophagy participates in regulating cardiac hypertrophy (Li et al. 2017b). Activation of autophagy is often thought to protect the heart, however, excessive autophagy induces cardiomyocyte atrophy and death (Schiattarella \& Hill 2016). Furthermore, autophagy disorders or reductions are in association with heart failure. Therefore, regulation of autophagy has become a therapeutic target for cardiovascular disease (Chen et al. 2016; Lin et al. 2016; Orogo \& Gustafsson 2015; Zhang et al. 2016b). It is critical to discover novel targets and related mechanisms to precise positioning. During autophagosome formation, the cytosolic microtubule-associated protein LC3-I is conjugated to phosphatidylethanolamine to produce LC3-II, which is recruited to the autophagosome membrane and degraded after autophagosome fusion to lysosomes. Hence, LC3-II/LC3-I ratio has been used to affect autophagy flux (Gu et al. 2016). Furthermore, Beclin-1 and p62 also have key roles in autophagy (Chen et al. 2016; Lin et al. 2016; Orogo \& Gustafsson 2015; Zhang et al. 2016b).

MicroRNAs (miRNAs) with about 22 nucleotides in length are small endogenous noncoding RNAs (Wang et al. 2012). MiRNAs can inhibit the expression of specific genes at the post-transcription level by binding to the 3'-untranslated region (3'-UTR) of the target mRNA (Wang et al. 2012). Many miRNAs have been confirmed to participate in cardiac hypertrophy (Tu et al. 2014; Verjans et al. 2017; Yang et al. 2016). It has been confirmed that many miRNAs are abnormally expressed in cardiac hypertrophy, which has the effect of promoting hypertrophy or resistance to hypertrophy (Jeong et al. 2012). These miRNAs could have potential value as therapeutic targets for heart disease. Therefore, it is of importance to explore the regulation mechanisms of miRNAs in cardiac hypertrophy. For instance, overexpressed miR-146a causes cardiac hypertrophy in vivo, while mR-146a knockout attenuates hypertrophic responses and heart dysfunctions (Heggermont et al. 2017). 
Growing evidence has confirmed that miR-26a-5p participates in a variety of diseases. For example, miR-26a defends vascular smooth muscle cells from $\mathrm{H}_{2} \mathrm{O}_{2}$-induced injury via activating PTEN/AKT /mTOR signaling axis (Peng et al. 2018). MiR-26a participates in regulating allergic inflammation (Kwon et al. 2015). Overexpressed miR-26a inhibits neuropathic pain and neuroinflammation in rat model with chronic sciatic nerve injury (Zhang et al. 2018). The roles of miR-26a in cardiovascular diseases have been pointed out in recent studies. For example, miR-26a can ameliorate the development of atherosclerosis by regulating TRPC3 (Feng et al. 2018). miR-26a can promote myocardial damage caused by myocardial ischemia and reperfusion (Gong et al. 2019). Up-regulation of miR-26a may promote myocardial fibrosis after acute myocardial infarction (Zhang \& Cui 2018). Yet, the function of miR-26a in cardiac hypertrophy has not been understood. In our study, we explored the regulatory function of miR-26a-5p in phenylephrine (PE)-induced cardiac hypertrophy and analyzed potential mechanisms. PE was employed to establish cardiac hypertrophy models. The results revealed that highly expressed miR-26a-5p promoted myocardial cell autophagy activation and cardiac hypertrophy in rat hearts by regulating GSK3 $\beta$.

\section{Materials and methods:}

\section{Cell culture and treatment}

H9C2 cells were purchased from the Cell Bank of Sai Lan Biological Technology Co., Ltd (Zhejiang, China). The cells were maintained in Dulbecco's Modified Eagle Medium (Invitrogen, Carlsbad, CA, USA) supplemented with 10\% fetal bovine serum (Invitrogen, Carlsbad, CA, USA) in a humidified atmosphere $\left(37^{\circ} \mathrm{C}, 5 \% \mathrm{CO}_{2}\right)$. The cells were treated by different concentrations of PE $(10 \mu \mathrm{M} ; 20 \mu \mathrm{M} ; 50 \mu \mathrm{M} ; 100 \mu \mathrm{M} ; 200 \mu \mathrm{M})$ for $48 \mathrm{~h}$. MiR-26a-5p agomir and inhibitor were used to transiently transfect H9C2 cells using Lipofectamine 2000. Lysosomal inhibitor Bafilomycin A1 (Baf-A1; Sangon Biotech; Shanghai, China) was dissolved to $100 \mu \mathrm{g} / \mathrm{ml}$ stock solution and diluted to $10 \mathrm{nM}$ to inhibit autophagy of $\mathrm{H} 9 \mathrm{C} 2$ cells.

\section{Transfection}

MiR-26a-5p agomir, miR-26a-5p inhibitor and corresponding controls were purchased from Genepharma (Shanghai, China). GSK3 $\beta$ full length (forward, 5'GAAGATTCTAGAGCTAGCGAATTCGCCACCATGTCGGGGCGACCGAGAAC-3'; reverse, 5'-GCGGCCGCGGATCCTCAGGTAGAGTTGGAGGCTGATG-3') and GSK3 $\beta$ siRNAs (siRNA1, 5'-GGAGAGCCCAATGTTTCAT3'; siRNA2, 5'CCGATTACACGTCTAGTAT-3'; siRNA3, 5'-GCTGTGTGTTGGCTGAATT-3') were designed and synthesized by General biosystems (Anhui, China). Transient transfection into H9C2 cells was performed using the Lipofectamine 2000.

\section{Quantitative real-time polymerase chain reaction (qRT-PCR)}

Total RNA was extracted from H9C2 cells or tissues using TRIzol reagent (Takara, Beijing, China). After that, the extracted RNA was reverse transcribed into cDNA using a RevertAid First Strand cDNA synthesis Kit (Thermo, Waltham, Massachusetts, USA). SYBR Green Master Mix (Takara, Beijing, China) was used for qRT-PCR. The PCR primer information of miR-26a-5p, 
$117 \mathrm{U} 6$, glycogen synthase kinase $3 \beta$ (GSK3 $\beta$ ), ANP, ACTA1, MYH7 and GAPDH is shown in 118 Table 1. U6 or GAPDH served as internal miRNA controls. The relative expression of target genes was determined by $2^{-\Delta \Delta \mathrm{Ct}}$ method.

\section{Western blot analysis}

H9C2 cells pretreated were divided into 6 groups including PE, miR-26a agomir, miR-26a agomir and PE, miR-26a inhibitor, miR-26a inhibitor and PE, and control group. H9C2 cells were lysed using RIPA buffer with a protease inhibitor cocktail (Sigma, NY, USA), which was separated using SDS-PAGE. After that, the protein was transferred onto PVDF membrane incubated with primary antibodies including anti-LC3 $(1: 1000,12741 \mathrm{~S}, \mathrm{CST}$, Danvers, MA,USA), anti-Beclin-1 (1:1000, CST), anti-p62 (1:1000, CST), anti- $\alpha$-actinin (1:200, abcam, UK), anti-GSK3 $\beta(1: 1000$, CST $)$ and anti-GAPDH $(1: 5000,2118 \mathrm{~L}, \mathrm{CST})$ overnight at $4{ }^{\circ} \mathrm{C}$ and then incubated with secondary antibody for $1 \mathrm{~h}$ at room temperature. Finally, ECL HRP Substrate Kit (Bio-Rad, Hercules, CA) was used to visualize the proteins. GAPDH served as an internal control.

\section{Immunofluorescence assay}

Treated $\mathrm{H} 9 \mathrm{C} 2$ cells were climbed in 24-well plates. On the second day, cells were transfected with miR-26a-5p agomir, miR-26a-5p inhibitor or the corresponding controls and treated with PE for $48 \mathrm{~h}$ for immunofluorescence staining. Then, the medium was removed and rinsed 3 times with $1 \times$ PBS. The cells were fixed with $4 \%$ paraformaldehyde at room temperature for $20 \mathrm{~min}$, and then $0.1 \%$ Triton X-100 solution was added at room temperature for $10 \mathrm{~min}$. The cells were blocked with $3 \%$ BSA solution at $37^{\circ} \mathrm{C}$ for $90 \mathrm{~min}$. After that, the cells were incubated with primary antibody including anti- $\alpha$-actinin (1:200, abcam, UK) or anti-LC3 (1:1000, CST, Danvers, MA, USA) at $4{ }^{\circ} \mathrm{C}$ overnight, followed by incubation with fluorescent secondary antibody $(1: 2000)$ at $37^{\circ} \mathrm{C}$ for $1.5 \mathrm{~h}$ in the dark. Then, the cells were stained with 4 ', 6-diamidino-2-phenylindole (DAPI) for $5 \mathrm{~min}$ at room temperature. After mounting, the cells were observed under a fluorescent microscope (ECLIPSE Ti-S; Nikon, Tokyo, Japan).

\section{Measurement of cell surface areas}

After climbing, the cells were fixed with 4\% paraformaldehyde for $15 \mathrm{~min}$, followed by permeation using $0.5 \%$ Triton X-100 at room temperature for $20 \mathrm{~min}$. Then, the cells were incubated with TRITC-phalloidin antibody (1:200; Shanghai Yisheng Biotechnology Co., Ltd., Shanghai, China) at room temperature in the dark for $30 \mathrm{~min}$. The cells were then incubated with DAPI in the dark for $5 \mathrm{~min}$. After washing away the excess DAPI using PBS, the liquid on the slide was absorbed with absorbent paper. The slide was mounted with the mounting solution containing anti-fluorescence quencher, and the images were observed under a fluorescent microscope.

\section{Luciferase reporter assay}

The 3'-UTR region of GSK3 $\beta$ containing amplified sites (wide type, $\mathrm{Wt}$ ) and mutant sites (mutant type, Mut) were conducted and cloned into pGL3-CM vectors (Ambion, Grand Island, NY, USA). Then, plasmids were co-transfected into H9C2 cells with miR-26a-5p agomir or NC using lipofectamine 2000 reagent (life technologies, Carlsbad, California, USA). Then, firefly 
158

159

160

161

162

163

164

165

166

167

168

169

170

171

172

173

174

luciferase activities were measured using Dual-Luciferase reporter assay system (Promega, Madison, WI, USA). Relative luciferase activity was normalized to Renilla luciferase activity. Animal experiments

Male spontaneously hypertensive rats (SHR; 12-weeks old; weighing 200-250g) were purchased from Shanghai Experimental Animal Center (Shanghai, China), which were used to construct a compensatory cardiac hypertrophy model. All rats were reared under 12/12 cycle of light at room temperature $\left(25-27^{\circ} \mathrm{C}\right)$ and fed with a regular diet. They were randomly divided into two groups: control $(n=4), N C$ agomir group $(n=4)$ and miR-26a-5p agomir group $(n=10)$. All rats were injected with miR-26a-5p agomir or NC agomir $(10 \mathrm{mg} / \mathrm{kg})$ in the tail vein. After continuous administration for 3 days, a total of 5 times, all rats were sacrificed with an overdose of $2 \%$ sodium pentobarbital. Fresh heart tissues were removed for further analysis. All animals were treated in accordance with the Guide for the Care and Use of Laboratory Animals. Our study was approved by the Ethics Committee of Zhejiang Province People's Hospital (20190232).

\section{Hematoxylin and eosin (H\&E)}

After harvesting and separating the hearts, the fresh heart tissues were fixed in 10\%

formalin solution for $24-48 \mathrm{~h}$, and then dehydrated by $70 \%, 80 \%, 90 \%, 95 \%, 100 \%$ ethanol I,

and $100 \%$ ethanol II for $1 \mathrm{~h}$ each time. Next, the tissues were treated with xylene I for $10 \mathrm{~min}$, xylene II for $20 \mathrm{~min}$, and then embedded in paraffin. The tissues were cut into $4 \mu \mathrm{m}$ thickness, and then placed in a $65^{\circ} \mathrm{C}$ box for 6-12h. H\&E staining was presented. Briefly, after the sections were dewaxed to water, the sections were immersed in hematoxylin staining solution at room temperature for $10 \mathrm{~min}$ and eosin staining solution for $5 \mathrm{~s}$. After dehydration, transparency, and sealing, the pathological changes of myocardial tissue were observed under a microscope (200×). Statistical analysis

Statistical analysis was carried out using GraphPad Prism 7.0 and SPSS 23. Results were presented as the mean \pm standard deviation, except for special. Differences between two groups were assessed by Student's $t$ test, while two-way analysis of variance (ANOVA) followed by Tukey post hoc testing was used for multiple comparisons. P-value $<0.05$ was considered statistically significant.

\section{Results:}

\section{MiR-26a-5p is highly expressed in PE-induced cardiac hypertrophy}

Firstly, we assessed whether the cardiac hypertrophy model was successfully built in vitro. H9C2 cells were treated with different concentrations of PE in vitro. qRT-PCR results showed that the expression of miR-26a-5p in $\mathrm{H} 9 \mathrm{C} 2$ cells was significantly elevated in PE-induced cardiac hypertrophy, with a dose-dependent manner (Figure 1A). After treatment with $200 \mu \mathrm{M} \mathrm{PE}$, miR26a-5p had the highest expression level in $\mathrm{H} 9 \mathrm{C} 2$ cells. Therefore, $200 \mu \mathrm{M}$ PE was used to conduct cardiac hypertrophy models. 
196

197

198

199

200

201

202

203

204

205

206

207

208

209

210

211

212

213

214

215

216

217

218

219

220

221

222

223

224

225

226

227

228

229

230

231

232

233

234

235

236

MiR-26a-5p promotes myocardial cell autophagy activation in PE-induced cardiac hypertrophy

Increasing evidence suggests that cardiomyocyte autophagy is closely related to cardiac hypertrophy. Thus, autophagy-related markers including LC3 (LC3-II and LC3-I), Beclin-1 and p62 were detected in H9C2 cells. We found that LC3II and Beclin-1 protein expression was markedly increased in PE-induced cardiac hypertrophy, while p62 protein expression was significantly decreased (Figure 1B, C). Intriguingly, miR-26a-5p agomir dramatically increased LC3II and Beclin-1 expression and decreased p62 expression in PE-induced H9C2 cells (Figure 1B-E). Instead, miR-26a-5p inhibitor significantly reversed PE-induced changes in Beclin-1 and p62 proteins (Figure 1D, E). In the presence or absence of lysosomal inhibitor Baf-A1, we compared LC3II and p62 expression levels in H9C2 cells. The results showed that miR-26a-5p agomir distinctly increased LC3II and decreased p62 expression in PE-induced H9C2 cells in the presence or absence of Baf-A1 (Figure 1F-H). Similar results were observed under immunofluorescence. miR-26a-5p agomir remarkably enhanced PE-induced increase in LC3II expression, on the contrary, miR-26a-5p inhibitor reversed PE-induced increase in LC3II expression (Figure 2A-S). Above results suggested that autophagy was activated in PE-induced cardiac hypertrophy.

Figure 1. MiR-26a promotes myocardial cell autophagy activation in PE-induced cardiac hypertrophy. (A) The mRNA expression levels of miR-26a-5p in H9C2 cells treated by different concentrations of PE. (B-E) Western blot analysis results showing the expression of LC3II, Beclin-1 and p62. (F-H) The expression of LC3II and p62 in H9C2 cells in the presence or absence of Baf-A1. Data were presented as mean $\pm \operatorname{SD}(n=3) .{ }^{*} p<0.05 ;{ }^{*} p<0.01$; $* * * * p<0.0001$.

Figure 2. Immunofluorescence results showing the effect of miR-26a-5p on LC3 protein expression in PE-induced cardiac hypertrophy. (A-R) Representative images of immunofluorescence results $(\times 200)$. (S) The expression of LC3 protein was measured in H9C2 cells treated with $200 \mu \mathrm{M}$ PE. Data were presented as mean $\pm \operatorname{SD}(n=3) .{ }^{*} \mathrm{p}<0.05$; $* * * * \mathrm{p}<0.0001$.

\section{MiR-26a-5p inhibits GSK3 $\beta$ expression and promotes cardiac hypertrophy in vitro}

To explore miR-26a-5p effects on cardiac hypertrophy, biomarker proteins related-to cardiac hypertrophy were tested. Firstly, we would confirm whether miR-26a-5p participated in regulating cardiac hypertrophy. qRT-PCR results revealed that the expression of miR-26a-5p in PE-induced cardiac hypertrophy was remarkably up-regulated compared with control group (Figure 3A). As expected, miR-26a-5p agomir significantly increased its expression in H9C2 cells treated with $200 \mu \mathrm{M}$ PE. Meanwhile, the expression of miR-26a-5p was remarkedly decreased in PE-induced H9C2 cells (Figure 3A). To further assess the function of miR-26a$5 \mathrm{p}$ in cardiac hypertrophy, we tested cardiac hypertrophy related marker protein GSK3 $\beta$ (a negative regulator of cardiac hypertrophy) expression. At the mRNA and protein levels, the expression of GSK3 $\beta$ was decreased in PE-induced cardiac hypertrophy compared to control group (Figure 3B-D). However, miR-26a-5p inhibitor significantly elevated the expression of GSK3 $\beta$ in H9C2 cells treated with PE (Figure 3B-D). Dual luciferase report results confirmed

Peer] reviewing PDF | (2019:10:41920:2:0:NEW 17 Oct 2020) 
237 the direct target relationship between miR-26a-5p and GSK3 $\beta$ 3'UTR. miR-26a-5p

238

239

240

241

242

243

244

245

246

247

248

249

250

251

252

253

254

255

256

257

258

259

260

261

262

263

264

265

266

267

268

269

270

271

272

273

274

275

276

277

overexpression could reduce luciferase activity of wild type GSK3 $\beta$, however such effects were not investigated for mutation type GSK3 $\beta$ (Figure 3E, F). According to above results, miR-26a$5 p$ inhibited antihypertrophic GSK3 $\beta$ expression in PE-induced cardiac hypertrophy in vitro.

The expression of cardiac hypertrophy-related protein $\alpha$-actinin was also examined in treated H9C2 cells. As shown in qRT-PCR results, the mRNA expression level of $\alpha$-actinin was remarkedly elevated in H9C2 cells after treatment with PE (Figure 3G). Also, PE-induced increase in $\alpha$-actinin expression was significantly strengthened by miR-26a-5p agomir, and was significantly decreased by miR-26a-5p inhibitor (Figure 3G). Similar results were investigated in immunofluorescence staining results (Figure 3H-Z). To further investigate the effect of miR26a-5p on cardiomyocyte surface areas, we performed TRITC-phalloidin fluorescence staining. Compared to control group, PE remarkedly promoted cardiomyocyte surface areas (Figure 4AS). As expected, miR-26a-5p agomir significantly increased PE-induced cardiomyocyte surface areas (Figure 4A-S). Instead, miR-26a-5p inhibitor reversed PE-induced cardiomyocyte surface areas (Figure 4A-S). These results revealed that miR-26a-5p promoted cardiac hypertrophy in vitro.

Figure 3. MiR-26a-5p inhibits GSK3 $\beta$ expression and promotes cardiac hypertrophy in vitro. (A, B) qRT-PCR results showing the expression levels of miR-26a-5p and GSK3 $\beta$ in H9C2 cells treated with PE. (C, D) Western blot showing the effect of miR-26a-5p on the expression of GSK3 $\beta$ and $\alpha$-actinin proteins. (E, F) Dual luciferase report results confirmed that miR-26a-5p could bind to GSK3 $\beta$ 3'UTR. (G) The effect of $\alpha$-actinin on the mRNA expression of $\alpha$-actinin according to qRT-PCR results. (H-Z) Immunofluorescence results showing the effect of miR-26a-5p on $\alpha$-actinin protein in PE-induced cardiac hypertrophy $(\times 200)$. The marker of $\alpha$-actinin was stained by green color. Data were presented as mean $\pm \operatorname{SD}(n=3)$. ${ }^{*} p<0.05$; $* * \mathrm{p}<0.01 ; * * * \mathrm{p}<0.001 ; * * * * \mathrm{p}<0.0001$.

Figure 4. Effects of miR-26a-5p on the surface areas in PE-induced cardiac hypertrophy. (A-R) Representative images of TRITC-phalloidin fluorescence staining. (S) Relative cardiomyocyte surface areas. Data were presented as mean $\pm \mathrm{SD}(\mathrm{n}=3)$. ${ }^{*} \mathrm{p}<0.05 ; * * \mathrm{p}<0.01$; $* * * * \mathrm{p}<0.0001$.

\section{miR-26a-5p promotes cardiac hypertrophy by regulating GSK3 $\beta$}

We further observed the effects of miR-26a-5p on cardiac hypertrophy-related markers including ANP, ACTA1 and MYH7. qRT-PCR results showed that the mRNA expression levels of ANP, ACTA1 and MYH7PE were significantly elevated in PE-induced cardiomyocytes (Figure 5A-C). PE-induced increase in cardiac hypertrophy-related markers was dramatically promoted by miR-26a-5p agomir and was reversed by its inhibitor (Figure 5A-C). To confirm that miR-26a-5p could promote cardiac hypertrophy by regulating GSK3 $\beta$, GSK3 $\beta$ was successfully overexpressed and silenced according to qRT-PCR results (Figure 5D, E). As shown in Figure 5F-H, GSK3 $\beta$ overexpression remarkedly decreased miR-26a-5p-induced increase in cardiac hypertrophy-related marker expression. Furthermore, silencing GSK3 $\beta$ significantly reversed miR-26a-5p inhibitor-induced decrease in cardiac hypertrophy-related marker expression in PE-induced H9C2 cells. Above results indicated that miR-26a-5p could

Peer) reviewing PDF | (2019:10:41920:2:0:NEW 17 Oct 2020) 
278

279

280

281

282

283

284

285

286

287

288

289

290

291

292

293

294

295

296

297

298

299

300

301

302

303

304

305

306

307

308

309

310

311

312

313

314

315

316

317

promote cardiac hypertrophy by regulating GSK3$\beta$.

Figure 5. miR-26a-5p promotes cardiac hypertrophy by regulating GSK3ß. (A-C) qRT-PCR results showing the effects of miR-26a-5p on PE-induced H9C2 cells. (D, E) GSK3 $\beta$ was successfully overexpressed and silenced in $\mathrm{H} 9 \mathrm{C} 2$ cells. (F-H) According to qRT-PCR results, miR-26a-5p could promote the expression levels of cardiac hypertrophy-related markers by regulating GSK3 $\beta$. Data were presented as mean $\pm \operatorname{SD}(n=3)$. ${ }^{* *} \mathrm{p}<0.01 ; * * * \mathrm{p}<0.001$; $* * * * \mathrm{p}<0.0001$.

\section{MiR-26a-5p inhibits GSK3 $\beta$ expression and promotes cardiac hypertrophy in vivo}

To confirm whether overexpressed miR-26a-5p promoted cardiac hypertrophy, miR-26a-5p agomir or NC agomir was injected into SHR. The results showed that miR-26a-5p expression was elevated in SHR injected miR-26a-5p agomir compared to control groups (Figure 6A). Additionally, we found that GSK3 $\beta$ mRNA and protein expression was both down-regulated in SHR hearts after injecting miR-26a-5p agomir compared to controls (Figure 6B, C). Furthermore, our western blot results showed that compared to control agomir group, LC3II and Beclin-1 expression was significantly elevated and p62 expression was significantly decreased in miR-26a-5p agomir group (Figure 6D-G). In Figure 6H-P, the cell surface area was increased in miR-26a-5p agomir group compared with control agomir group. qRT-PCR showed that cardiac hypertrophy-related markers including ANP, ACTA1 and MYH7 were significantly elevated in miR-26a-5p agomir group compared to controls (Figure 6Q-S). Therefore, above results suggested that miR-26a-5p inhibited GSK $3 \beta$ expression and promoted cardiac hypertrophy in vivo.

Figure 6. MiR-26a-5p inhibits GSK3 $\beta$ expression and promotes cardiac hypertrophy in vivo. (A, B) qRT-PCR showing the mRNA expression levels of miR-26a-5p and GSK3 $\beta$ in SHR injected with miR-26a agomir. (C-G) The protein expression levels of GSK3 $\beta$, LC3II, Beclin-1 and 62 were detected in myocardial tissues by western blot. (H-P) Hematoxylin and eosin (H\&E) staining of myocardial tissues in the three groups $(\times 200)$. (Q-S) qRT-PCR results showing the mRNA expression levels of cardiac hypertrophy-related markers including ANP, ACTA1 and MYH7 in myocardial tissues. Data were presented as mean $\pm \mathrm{SD}(\mathrm{n}=3) .{ }^{* *} \mathrm{p}$-value $<0.01 ; * * * *$ p-value $<0.0001 ;$ ns: no statistical significance.

\section{Discussion:}

It has been confirmed that pathological hypertrophy may result in increased interstitial fibrosis, cell death and cardiac dysfunction (Dong et al. 2018). As a hallmark of cardiac ageing, cardiac hypertrophy may induce an increased incidence of cardiac disease (Woodall \& Gustafsson 2018), which maintains cardiac homeostasis in a physiological environment (Bernardo et al. 2010). Due to the limited ability of adult myocardium to regenerate, the loss of functional cardiomyocytes caused by autophagy is one of the potential mechanisms of myocardial remodeling and cardiac disease (Sun et al. 2018). In our study, miR-26a-5p was upregulated in PE-induced cardiac hypertrophy, furthermore, high miR-26a expression promoted myocardial cell autophagy activation and cardiac hypertrophy by GSK3 $\beta$ (Gu et al. 2016). 
318

319

320

321

322

323

324

325

326

327

328

329

330

331

332

333

334

335

336

337

338

339

340

341

342

343

344

345

346

347

348

349

350

351

352

353

354

355

356

357

358

As an endogenous regulator, miRNA participates in mediating post-transcriptional gene expression (Sadiq et al. 2017), while cardiac hypertrophy is largely dependent on gene expression (Qi et al. 2019). Therefore, there is reason to believe that miRNAs participate in cardiac hypertrophy (Gupta \& Thum 2016). For example, miR-199a inhibits autophagy and contributes to cardiac hypertrophy ( $\mathrm{Li}$ et al. 2017c). MiR-181a has been confirmed to be highly expressed in Ang II-induced cardiac hypertrophy and mediate autophagy (Li et al. 2017a). MiR365 induces cardiac hypertrophy and inhibits autophagy through mediating Skp2 expression (Wu et al. 2017). MiR-208a-3p activates autophagy in Ang II-induced cardiac hypertrophy via PDCD4-ATG5 pathway (Wang et al. 2018). MiR-22 also has been found to be a key regulatory factor of cardiac autophagy (Gupta et al. 2016). Yet, the function of miR-26a-5p in cardiac hypertrophy remains unclear. In our study, we successfully constructed cardiac hypertrophy models in vitro and in vivo. MiR-26a-5p was up-regulated in PE-induced cardiac hypertrophy. It has been found that LC3, Beclin-1 and p62 are key regulators of autophagy, and reversion of their expression could attenuate cardiac hypertrophy (Noh et al. 2016; Pan et al. 2017; Xie et al. 2018). Therefore, the expression levels of LC3, Beclin-1 and p62 can reflect the autophagy in cardiac hypertrophy. In our study, after PE-induced cardiac hypertrophy cells were treated by miR-26a-5p agomir, the expression of LC3II and Beclin-1 proteins was increased and p62 protein expression was decreased in the presence or absence of lysosomal inhibitor, suggesting that high miR-26a-5p expression may promote autophagy in cardiac hypertrophy.

GSK3 $\beta$ regulates various cellular functions by phosphorylating cellular substrates (Hardt \& Sadoshima 2002). The activation of GSK-3 $\beta$ is inhibited through PKB / Akt and Wnt signaling pathways. Growing studies suggest that GSK-3 $\beta$ negatively regulates cardiac hypertrophy, and inhibits GSK3 $\beta$ via hypertrophic stimulation contributes to cardiac hypertrophy. Because of its phosphorylatio, inhibition of GSK3 $\beta$ is associated with cardiac hypertrophy in response to endothelin-1 or phenylephrine ( $\mathrm{Li}$ et al. 2007; Markou et al. 2008). Our results revealed that GSK3 $\beta$ expression was inhibited in PE-induced cardiac hypertrophy treated by miR-26a agomir, which was consistent with previous studies. More importantly, dual luciferase report results confirmed that miR-26a-5p could bind to GSK3 $\beta$ 3'UTR. miR-26a-5p dramatically promoted PE-induced increase in cardiac hypertrophy-related markers including ANP, ACTA1 and MYH7. GSK3 $\beta$ could remarkedly reversed miR-26a-5p-induced increase in cardiac hypertrophyrelated marker expression. Thus, our findings revealed that miR-26a-5p could promote cardiac hypertrophy by regulating GSK3$\beta$.

It has been confirmed that $\alpha$-actinin is up-regulated in cardiac hypertrophy, which has been a hallmark of cardiac hypertrophy. Angiotensin II promotes $\alpha$-actinin expression in cardiac fibroblasts (Kawano et al. 2000; Sheng et al. 2016). In our study, qRT-PCR and immunofluorescence staining results demonstrated that $\alpha$-actinin expression was increased in PE-induced cardiac hypertrophy transfected by miR-26a-5p agomir. Therefore, overexpressed miR-26-5p induced cardiac hypertrophy by promoting $\alpha$-actinin expression. To further confirm whether overexpression of miR-26a-5p could contribute to cardiac hypertrophy, we measured cell surface area. We found that cell surface area was significantly increased in H9C2 cells transfected by miR-26a-5p agomir, which confirmed our conclusion. 
359

360

361

362

363

364

365

366

367

368

369

370

371

372

373

374

375

376

377

378

379

380

381

382

383

384

385

386

387

388

389

390

391

392

393

394

395

396

In addition, we successfully established cardiac hypertrophy models in vivo. qRT-PCR analysis suggested that GSK3 $\beta$ expression was decreased in SHR transfected by miR-26a agomir. As shown in H\&E staining results, overexpressed miR-26a-5p could aggravate cardiac hypertrophy. Furthermore, miR-26a-5p agomir significantly promoted cardiac hypertrophyrelated markers including ANP, ACTA1 and MYH7. Therefore, high miR-26a-5p expression inhibited GSK3 $\beta$ expression and promoted cardiac hypertrophy in vivo.

\section{Conclusion:}

Our findings reveal that miR-26a-5p promotes PE-induced cardiac hypertrophy by regulating GSK3$\beta$ and activates autophagy in vitro and in vivo, which may provide a novel insight into the pathogenesis of cardiac hypertrophy.

\section{Abbreviations:}

miRNAs: microRNAs; PE: phenylephrine; qRT-PCR: quantitative real-time polymerase chain reaction; SHR: spontaneously Hypertensive Rats; H\&E: Haematoxylin-Eosin; GSK3ß: glycogen

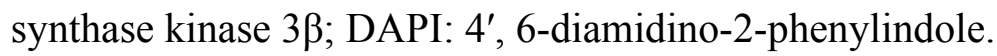

\section{Declarations:}

1. Acknowledgements

Not applicable.

2. Funding

This work was funded by Zhejiang Analytical Testing Fund (2017C37068) and Zhejiang

Medical and Health Research Fund (2018KY259).

3. Availability of data and material

The datasets supporting the conclusions of this article are included within the article (and its additional files).

4. Authors' contributions

Liqun Tang conceived and designed the study. Jianhong Xie, Xiaoqin Yu conducted most of the experiments and data analysis, and wrote the manuscript. Yangyang Zheng participated in collecting data and helped to draft the manuscript. All authors reviewed and approved the manuscript.

5. Ethics approval and consent to participate

The study was approved by the Ethics Committee of Zhejiang Province People's Hospital (20190232).

6. Consent for publication

Not applicable.

7. Conflicts of Interest

The authors declare no conflicts of interest. 


\section{References:}

399

400

401

402

403

404

405

406

407

408

409

410

411

412

413

414

415

416

417

418

419

420

421

422

423

424

425

426

427

428

429

430

431

432

433

434

435

436

Bernardo BC, Weeks KL, Pretorius L, and McMullen JR. 2010. Molecular distinction between physiological and pathological cardiac hypertrophy: experimental findings and therapeutic strategies. Pharmacol Ther 128:191-227. 10.1016/j.pharmthera.2010.04.005

Chen Q, Zhang L, Chen S, Huang Y, Li K, Yu X, Wu H, Tian X, Zhang C, Tang C, Du J, and Jin H. 2016. Downregulated endogenous sulfur dioxide/aspartate aminotransferase pathway is involved in angiotensin II-stimulated cardiomyocyte autophagy and myocardial hypertrophy in mice. Int J Cardiol 225:392-401. 10.1016/j.ijcard.2016.09.111

Dong Y, Liu C, Zhao Y, Ponnusamy M, Li P, and Wang K. 2018. Role of noncoding RNAs in regulation of cardiac cell death and cardiovascular diseases. Cell Mol Life Sci 75:291300. 10.1007/s00018-017-2640-8

Feng M, Xu D, and Wang L. 2018. miR-26a inhibits atherosclerosis progression by targeting TRPC3. Cell Biosci 8:4. 10.1186/s13578-018-0203-9

Gong DD, Yu J, Yu JC, and Jiang XD. 2019. Effect of miR-26a targeting GSK-3 $/ \beta$-catenin signaling pathway on myocardial apoptosis in rats with myocardial ischemia-reperfusion. Eur Rev Med Pharmacol Sci 23:7073-7082. 10.26355/eurrev_201908_18751

$\mathrm{Gu}$ J, Hu W, Song ZP, Chen YG, Zhang DD, and Wang CQ. 2016. Rapamycin Inhibits Cardiac Hypertrophy by Promoting Autophagy via the MEK/ERK/Beclin-1 Pathway. Front Physiol 7:104. 10.3389/fphys.2016.00104

Gupta SK, Foinquinos A, Thum S, Remke J, Zimmer K, Bauters C, de Groote P, Boon RA, de Windt LJ, Preissl S, Hein L, Batkai S, Pinet F, and Thum T. 2016. Preclinical Development of a MicroRNA-Based Therapy for Elderly Patients With Myocardial Infarction. J Am Coll Cardiol 68:1557-1571. 10.1016/j.jacc.2016.07.739

Gupta SK, and Thum T. 2016. Non-coding RNAs as orchestrators of autophagic processes. $J$ Mol Cell Cardiol 95:26-30. 10.1016/j.yjmcc.2015.11.012

Hardt SE, and Sadoshima J. 2002. Glycogen synthase kinase-3beta: a novel regulator of cardiac hypertrophy and development. Circ Res 90:1055-1063. 10.1161/01.res.0000018952.70505.f1

Heggermont WA, Papageorgiou AP, Quaegebeur A, Deckx S, Carai P, Verhesen W, Eelen G, Schoors S, van Leeuwen R, Alekseev S, Elzenaar I, Vinckier S, Pokreisz P, Walravens AS, Gijsbers R, Van Den Haute C, Nickel A, Schroen B, van Bilsen M, Janssens S, Maack C, Pinto Y, Carmeliet P, and Heymans S. 2017. Inhibition of MicroRNA-146a and Overexpression of Its Target Dihydrolipoyl Succinyltransferase Protect Against Pressure Overload-Induced Cardiac Hypertrophy and Dysfunction. Circulation 136:747761. 10.1161/circulationaha.116.024171

Jeong MH, Lee JS, Kim DH, Park WJ, and Yang DK. 2012. Identification of novel microRNAs negatively regulating cardiac hypertrophy. Biochem Biophys Res Commun 428:191-196. 10.1016/j.bbrc.2012.10.040

Kawano H, Cody RJ, Graf K, Goetze S, Kawano Y, Schnee J, Law RE, and Hsueh WA. 2000. 
Angiotensin II enhances integrin and alpha-actinin expression in adult rat cardiac fibroblasts. Hypertension 35:273-279. 10.1161/01.hyp.35.1.273

Kwon Y, Kim Y, Eom S, Kim M, Park D, Kim H, Noh K, Lee H, Lee YS, Choe J, Kim YM, and Jeoung D. 2015. MicroRNA-26a/-26b-COX-2-MIP-2 Loop Regulates Allergic Inflammation and Allergic Inflammation-promoted Enhanced Tumorigenic and Metastatic Potential of Cancer Cells. J Biol Chem 290:14245-14266. 10.1074/jbc.M115.645580

Li AL, Lv JB, and Gao L. 2017a. MiR-181a mediates Ang II-induced myocardial hypertrophy by mediating autophagy. Eur Rev Med Pharmacol Sci 21:5462-5470. 10.26355/eurrev_201712_13936

Li HJ, Yin H, Yao YY, Shen B, Bader M, Chao L, and Chao J. 2007. Tissue kallikrein protects against pressure overload-induced cardiac hypertrophy through kinin B2 receptor and glycogen synthase kinase-3beta activation. Cardiovasc Res 73:130-142. 10.1016/j.cardiores.2006.10.014

Li T, Fan J, Wang B, Traugh N, Chen Q, Liu JS, Li B, and Liu XS. 2017b. TIMER: A Web Server for Comprehensive Analysis of Tumor-Infiltrating Immune Cells. Cancer Res 77:e108-e110. 10.1158/0008-5472.Can-17-0307

Li Z, Song Y, Liu L, Hou N, An X, Zhan D, Li Y, Zhou L, Li P, Yu L, Xia J, Zhang Y, Wang J, and Yang X. 2017c. miR-199a impairs autophagy and induces cardiac hypertrophy through mTOR activation. Cell Death Differ 24:1205-1213. 10.1038/cdd.2015.95

Lin L, Liu X, Xu J, Weng L, Ren J, Ge J, and Zou Y. 2016. Mas receptor mediates cardioprotection of angiotensin-(1-7) against Angiotensin II-induced cardiomyocyte autophagy and cardiac remodelling through inhibition of oxidative stress. J Cell Mol Med 20:48-57. 10.1111/jcmm. 12687

Markou T, Cullingford TE, Giraldo A, Weiss SC, Alsafi A, Fuller SJ, Clerk A, and Sugden PH. 2008. Glycogen synthase kinases 3alpha and 3beta in cardiac myocytes: regulation and consequences of their inhibition. Cell Signal 20:206-218. 10.1016/j.cellsig.2007.10.004

Noh HS, Hah YS, Zada S, Ha JH, Sim G, Hwang JS, Lai TH, Nguyen HQ, Park JY, Kim HJ, Byun JH, Hahm JR, Kang KR, and Kim DR. 2016. PEBP1, a RAF kinase inhibitory protein, negatively regulates starvation-induced autophagy by direct interaction with LC3. Autophagy 12:2183-2196. 10.1080/15548627.2016.1219013

Orogo AM, and Gustafsson AB. 2015. Therapeutic targeting of autophagy: potential and concerns in treating cardiovascular disease. Circ Res 116:489-503. 10.1161/circresaha.116.303791

Pan Y, Qian JX, Lu SQ, Chen JW, Zhao XD, Jiang Y, Wang LH, and Zhang GX. 2017. Protective effects of tanshinone IIA sodium sulfonate on ischemia-reperfusion-induced myocardial injury in rats. Iran J Basic Med Sci 20:308-315. 10.22038/ijbms.2017.8361

Peng J, He X, Zhang L, and Liu P. 2018. MicroRNA26a protects vascular smooth muscle cells against $\mathrm{H} 2 \mathrm{O} 2$ induced injury through activation of the PTEN/AKT/mTOR pathway. Int $J$ Mol Med 42:1367-1378. 10.3892/ijmm.2018.3746

Qi H, Ren J, E M, Zhang Q, Cao Y, Ba L, Song C, Shi P, Fu B, and Sun H. 2019. MiR-103 
478

479

480

481

482

483

484

485

486

487

488

489

490

491

492

493

494

495

496

497

498

499

500

501

502

503

504

505

506

507

508

509

510

511

512

513

514

515

516

517

518

inhibiting cardiac hypertrophy through inactivation of myocardial cell autophagy via targeting TRPV3 channel in rat hearts. J Cell Mol Med 23:1926-1939.

$10.1111 / \mathrm{jcmm} .14095$

Sadiq S, Crowley TM, Charchar FJ, Sanigorski A, and Lewandowski PA. 2017. MicroRNAs in a hypertrophic heart: from foetal life to adulthood. Biol Rev Camb Philos Soc 92:13141331. 10.1111/brv.12283

Schiattarella GG, and Hill JA. 2016. Therapeutic targeting of autophagy in cardiovascular disease. J Mol Cell Cardiol 95:86-93. 10.1016/j.yjmcc.2015.11.019

Sheng JJ, Feng HZ, Pinto JR, Wei H, and Jin JP. 2016. Increases of desmin and alpha-actinin in mouse cardiac myofibrils as a response to diastolic dysfunction. J Mol Cell Cardiol 99:218-229. 10.1016/j.yjmcc.2015.10.035

Sun T, Li MY, Li PF, and Cao JM. 2018. MicroRNAs in Cardiac Autophagy: Small Molecules and Big Role. Cells 7. 10.3390/cells7080104

Tang X, Chen XF, Wang NY, Wang XM, Liang ST, Zheng W, Lu YB, Zhao X, Hao DL, Zhang ZQ, Zou MH, Liu DP, and Chen HZ. 2017. SIRT2 Acts as a Cardioprotective Deacetylase in Pathological Cardiac Hypertrophy. Circulation 136:2051-2067. 10.1161/circulationaha.117.028728

Tu Y, Wan L, Zhao D, Bu L, Dong D, Yin Z, Cheng Z, and Shen B. 2014. In vitro and in vivo direct monitoring of miRNA-22 expression in isoproterenol-induced cardiac hypertrophy by bioluminescence imaging. Eur J Nucl Med Mol Imaging 41:972-984. 10.1007/s00259013-2596-3

Verjans R, van Bilsen M, and Schroen B. 2017. MiRNA Deregulation in Cardiac Aging and Associated Disorders. Int Rev Cell Mol Biol 334:207-263. 10.1016/bs.ircmb.2017.03.004

Wang J, Song Y, Zhang Y, Xiao H, Sun Q, Hou N, Guo S, Wang Y, Fan K, Zhan D, Zha L, Cao Y, Li Z, Cheng X, Zhang Y, and Yang X. 2012. Cardiomyocyte overexpression of miR$27 \mathrm{~b}$ induces cardiac hypertrophy and dysfunction in mice. Cell Res 22:516-527. 10.1038/cr.2011.132

Wang L, Ye N, Lian X, Peng F, Zhang H, and Gong H. 2018. MiR-208a-3p aggravates autophagy through the PDCD4-ATG5 pathway in Ang II-induced H9c2 cardiomyoblasts. Biomed Pharmacother 98:1-8. 10.1016/j.biopha.2017.12.019

Woodall BP, and Gustafsson AB. 2018. Autophagy-A key pathway for cardiac health and longevity. Acta Physiol (Oxf) 223:e13074. 10.1111/apha.13074

Wu H, Wang Y, Wang X, Li R, and Yin D. 2017. MicroRNA-365 accelerates cardiac hypertrophy by inhibiting autophagy via the modulation of Skp2 expression. Biochem Biophys Res Commun 484:304-310. 10.1016/j.bbrc.2017.01.108

Xie YP, Lai S, Lin QY, Xie X, Liao JW, Wang HX, Tian C, and Li HH. 2018. CDC20 regulates cardiac hypertrophy via targeting LC3-dependent autophagy. Theranostics 8:5995-6007. 10.7150/thno. 27706

Yang L, Li Y, Wang X, Mu X, Qin D, Huang W, Alshahrani S, Nieman M, Peng J, Essandoh K, Peng T, Wang Y, Lorenz J, Soleimani M, Zhao ZQ, and Fan GC. 2016. Overexpression of miR-223 Tips the Balance of Pro- and Anti-hypertrophic Signaling Cascades toward 
519

520

521

522

523

524

525

526

527

528

529

530

531

532

533

534

535

536

537

538

539

540

541

542

543

544

545

546

547

548

549

550

551

552

553

554

555

556

557

558

Physiologic Cardiac Hypertrophy. J Biol Chem 291:15700-15713. 10.1074/jbc.M116.715805

Yang Y, Del Re DP, Nakano N, Sciarretta S, Zhai P, Park J, Sayed D, Shirakabe A, Matsushima S, Park Y, Tian B, Abdellatif M, and Sadoshima J. 2015. miR-206 Mediates YAPInduced Cardiac Hypertrophy and Survival. Circ Res 117:891-904. 10.1161/circresaha.115.306624

Zhang H, Li S, Zhou Q, Sun Q, Shen S, Zhou Y, Bei Y, and Li X. 2016a. Qiliqiangxin Attenuates Phenylephrine-Induced Cardiac Hypertrophy through Downregulation of MiR-199a-5p. Cell Physiol Biochem 38:1743-1751. 10.1159/000443113

Zhang S, and Cui R. 2018. The targeted regulation of miR-26a on PTEN-PI3K/AKT signaling pathway in myocardial fibrosis after myocardial infarction. Eur Rev Med Pharmacol Sci 22:523-531. 10.26355/eurrev_201801_14205

Zhang X, Gibson ME, Li ZL, Zhu XY, Jordan KL, Lerman A, and Lerman LO. 2016 b. Autophagy Portends the Level of Cardiac Hypertrophy in Experimental Hypertensive Swine Model. Am J Hypertens 29:81-89. 10.1093/ajh/hpv057

Zhang Y, Su Z, Liu HL, Li L, Wei M, Ge DJ, and Zhang ZJ. 2018. Effects of miR-26a-5p on neuropathic pain development by targeting MAPK6 in in CCI rat models. Biomed Pharmacother 107:644-649. 10.1016/j.biopha.2018.08.005

\section{Figure legends:}

Figure 1. MiR-26a promotes myocardial cell autophagy activation in PE-induced cardiac hypertrophy. (A) The mRNA expression levels of miR-26a-5p in H9C2 cells treated by different concentrations of PE. (B-E) Western blot analysis results showing the expression of LC3II, Beclin-1 and p62. (F-H) The expression of LC3II and p62 in H9C2 cells in the presence or absence of Baf-A1. Data were presented as mean $\pm \operatorname{SD}(n=3) .{ }^{*} \mathrm{p}<0.05 ;{ }^{*} \mathrm{p}<0.01$; $* * * * \mathrm{p}<0.0001$.

Figure 2. Immunofluorescence results showing the effect of miR-26a-5p on LC3 protein expression in PE-induced cardiac hypertrophy. (A-R) Representative images of immunofluorescence results $(\times 200)$. (S) The expression of LC3 protein was measured in H9C2 cells treated with $200 \mu \mathrm{M}$ PE. Data were presented as mean $\pm \operatorname{SD}(n=3) .{ }^{*} \mathrm{p}<0.05$; $* * * * \mathrm{p}<0.0001$

Figure 3. MiR-26a-5p inhibits GSK3 $\beta$ expression and promotes cardiac hypertrophy in vitro. (A, B) qRT-PCR results showing the expression levels of miR-26a-5p and GSK3 $\beta$ in H9C2 cells treated with PE. (C, D) Western blot showing the effect of miR-26a-5p on the expression of GSK $3 \beta$ and $\alpha$-actinin proteins. (E, F) Dual luciferase report results confirmed that miR-26a-5p could bind to GSK3 $\beta$ 3'UTR. (G) The effect of $\alpha$-actinin on the mRNA expression of $\alpha$-actinin according to qRT-PCR results. (H-Z) Immunofluorescence results showing the effect of miR$26 a-5 p$ on $\alpha$-actinin protein in PE-induced cardiac hypertrophy $(\times 200)$. The marker of $\alpha$-actinin was stained by green color. Data were presented as mean $\pm \operatorname{SD}(n=3) .{ }^{*} \mathrm{p}<0.05 ; * * \mathrm{p}<0.01$; $* * * \mathrm{p}<0.001 ; * * * * \mathrm{p}<0.0001$. 
559 Figure 4. Effects of miR-26a-5p on the surface areas in PE-induced cardiac hypertrophy. (A-R) 560 Representative images of TRITC-phalloidin fluorescence staining. (S) Relative cardiomyocyte 561 surface areas. Data were presented as mean $\pm \mathrm{SD}(\mathrm{n}=3) .{ }^{*} \mathrm{p}<0.05 ;{ }^{* *} \mathrm{p}<0.01 ; * * * * \mathrm{p}<0.0001$. 562 Figure 5. miR-26a-5p promotes cardiac hypertrophy by regulating GSK3 $\beta$. (A-C) qRT-PCR 563 results showing the effects of miR-26a-5p on PE-induced H9C2 cells. (D, E) GSK3 $\beta$ was 564 successfully overexpressed and silenced in $\mathrm{H} 9 \mathrm{C} 2$ cells. (F-H) According to qRT-PCR results, 565 miR-26a-5p could promote the expression levels of cardiac hypertrophy-related markers by 566 regulating GSK3 3 . Data were presented as mean $\pm \operatorname{SD}(\mathrm{n}=3) .{ }^{* *} \mathrm{p}<0.01 ; * * * \mathrm{p}<0.001$; $567 * * * * \mathrm{p}<0.0001$.

568 Figure 6. MiR-26a-5p inhibits GSK3 $\beta$ expression and promotes cardiac hypertrophy in vivo. (A, 569 B) qRT-PCR showing the mRNA expression levels of miR-26a-5p and GSK3 $\beta$ in SHR injected 570 with miR-26a agomir. (C-G) The protein expression levels of GSK3 $\beta$, LC3II, Beclin-1 and p62 571 were detected in myocardial tissues by western blot. (H-P) Hematoxylin and eosin (H\&E) 572 staining of myocardial tissues in the three groups $(\times 200)$. (Q-S) qRT-PCR results showing the 573 mRNA expression levels of cardiac hypertrophy-related markers including ANP, ACTA1 and 574 MYH7 in myocardial tissues. Data were presented as mean $\pm \operatorname{SD}(n=3)$. **p-value $<0.01 ; * * * * p-$ 575 value $<0.0001$; ns: no statistical significance.

576 Table 1. The sequence of primers for qRT-PCR. 
Table $\mathbf{1}$ (on next page)

The sequence of primers for qRT-PCR. 
1 Table 1 . The sequence of primers for qRT-PCR.

\begin{tabular}{ll}
\hline Target & Sequence (5'-3') \\
\hline Rat miR-26a-5p & 5'-ACACTCCAGCTGGGTTCAAGTAATCCAGGA-3'(forward) \\
& 5'- \\
& CTCAACTGGTGTCGTGGAGTCGGCAATTCAGTTGAGAG \\
& CCTATC-3'(reverse) \\
Rat U6 & 5'-CTCGCTTCGGCAGCACA-3'(forward) \\
& 5'-AACGCTTCACGAATTTGCGT-3'(reverse) \\
Rat GSK3 $\beta$ & 5'-AAGCCCAGCCTACTAACAACC-3'(forward) \\
& 5'-CAGCCCCACTGTACTGACTG-3'(reverse) \\
Rat ANP & 5'-GGGCTTCTTCCTCTTCCTG-3'(forward) \\
Rat ACTA1 & 5'-CGCTTCATCGGTCTGCTC-3'(reverse) \\
& 5'-CTCTTGTGTGTGACAACGGC-3'(forward) \\
Rat MYH7 & 5'-CCCATACCGACCATGACACC-3'(reverse) \\
& 5'-TCAGTCATGGCGGATCGAG-3'(forward) \\
Rat GAPDH & 5'-ACAGTCACCGTCTTGCCATT-3'(reverse) \\
& 5'-GGAGCGAGATCCCTCCAAAAT-3'(forward) \\
Rat GSK3 $\beta$ & 5'-GGCTGTTGTCATACTTCTCATGG-3'(reverse) \\
& 5'-GAGACACACCTGCCCTCTTC-3'(forward) \\
Rat GAPDH & 5'-TGGGGCTGTTCAGGTAGAGT-3'(reverse) \\
& 5'-GCAAGTTCAACGGCACAG-3'(forward) \\
& 5'-GCCAGTAGACTCCACGACAT-3'(reverse) \\
\hline
\end{tabular}

2 
Figure 1

MiR-26a promotes myocardial cell autophagy activation in PE-induced cardiac hypertrophy.

(A) The mRNA expression levels of miR-26a-5p in H9C2 cells treated by different concentrations of PE. (B-E) Western blot analysis results showing the expression of LCZII, Beclin-1 and p62. (F-H) The expression of LC3II and p62 in H9C2 cells in the presence or absence of Baf-A1. Data were presented as mean $\pm S D(n=3)$. ${ }^{*}<<0.05 ; * *<<0.01$; $* * * * p<0.0001$. 

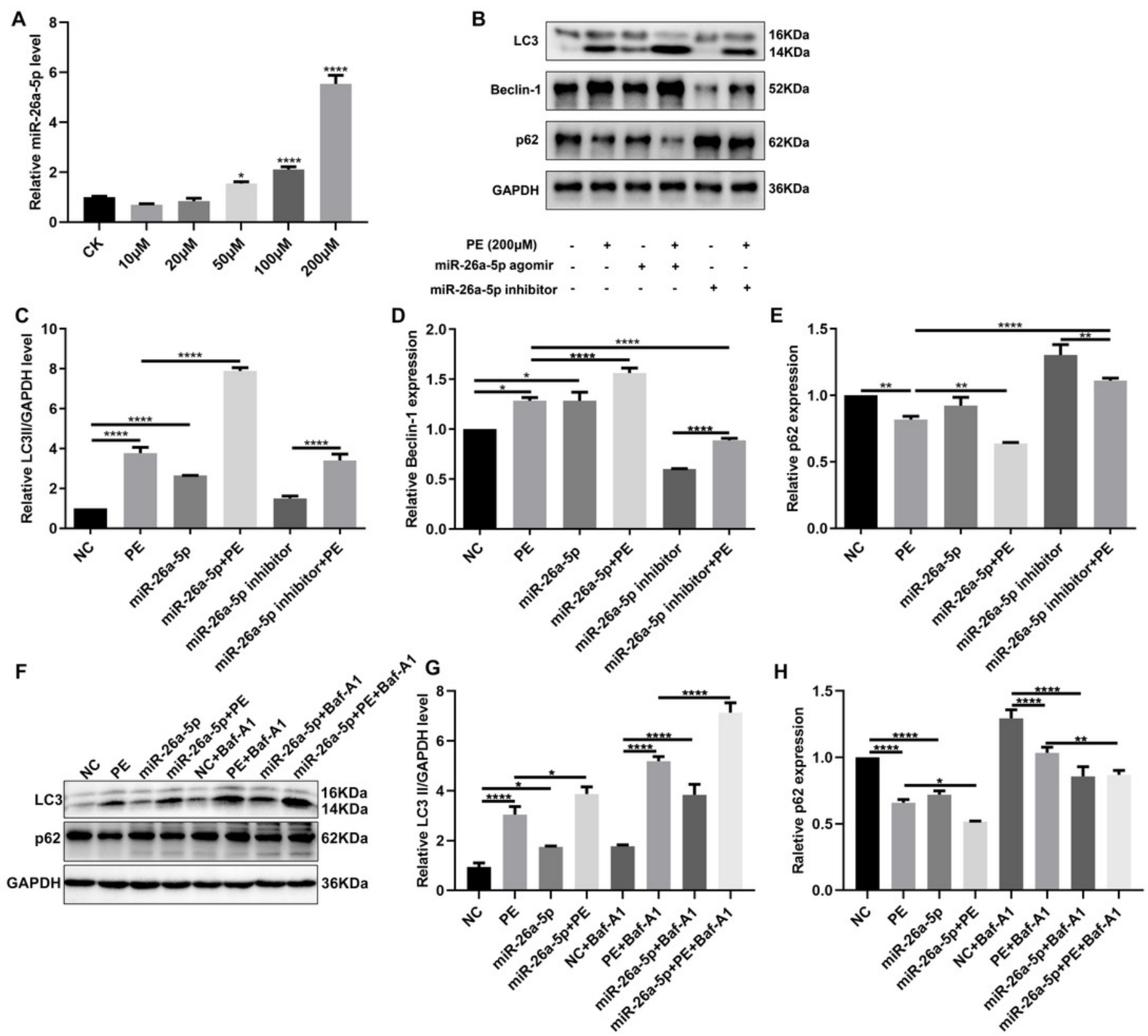
Figure 2

Immunofluorescence results showing the effect of miR-26a-5p on LC3 protein expression in PE-induced cardiac hypertrophy.

(A-R) Representative images of immunofluorescence results $(\times 200)$. (S) The expression of LC3 protein was measured in H9C2 cells treated with $200 \mu \mathrm{M}$ PE. Data were presented as mean \pm SD $(n=3) . * p<0.05 ; * * * * p<0.0001$.
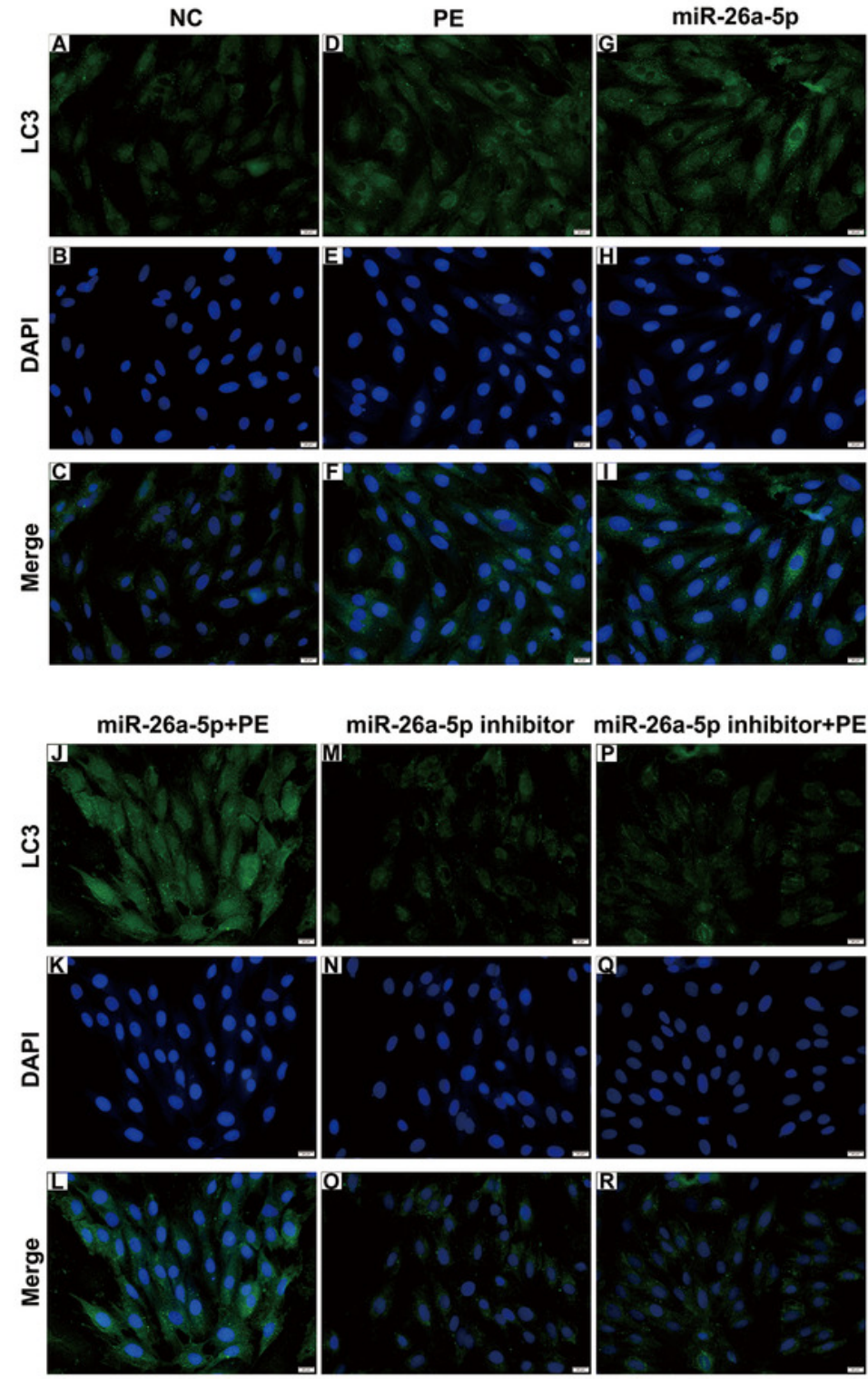

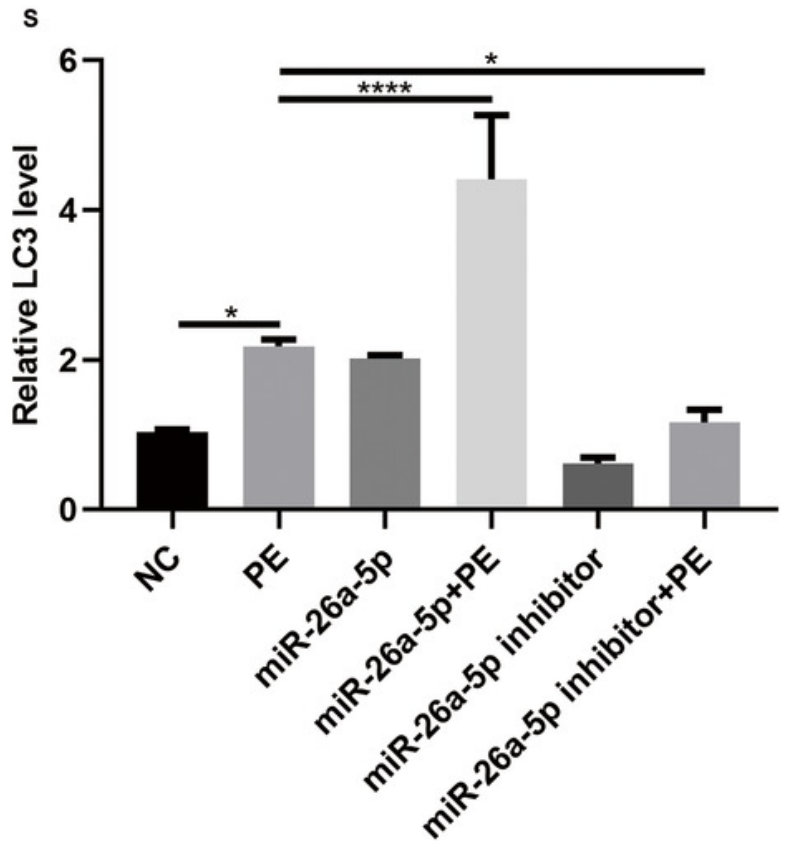




\section{Figure 3}

MiR-26a-5p inhibits GSK3 $\beta$ expression and promotes cardiac hypertrophy in vitro.

(A, B) qRT-PCR results showing the expression levels of miR-26a-5p and GSK3B in H9C2 cells treated with PE. (C, D) Western blot showing the effect of miR-26a-5p on the expression of GSK3 $\beta$ and $\alpha$-actinin proteins. (E, F) Dual luciferase report results confirmed that miR-26a-5p could bind to GSK3 $\beta$ 3'UTR. (G) The effect of $\alpha$-actinin on the mRNA expression of $\alpha$-actinin according to qRT-PCR results. (H-Z) Immunofluorescence results showing the effect of miR-26a-5p on $\alpha$-actinin protein in PE-induced cardiac hypertrophy $(\times 200)$. The marker of $\alpha$ actinin was stained by green color. Data were presented as mean $\pm S D(n=3)$. ${ }^{*}<<0.05$; $* * p<0.01 ; * * * p<0.001 ; * * * * p<0.0001$. 

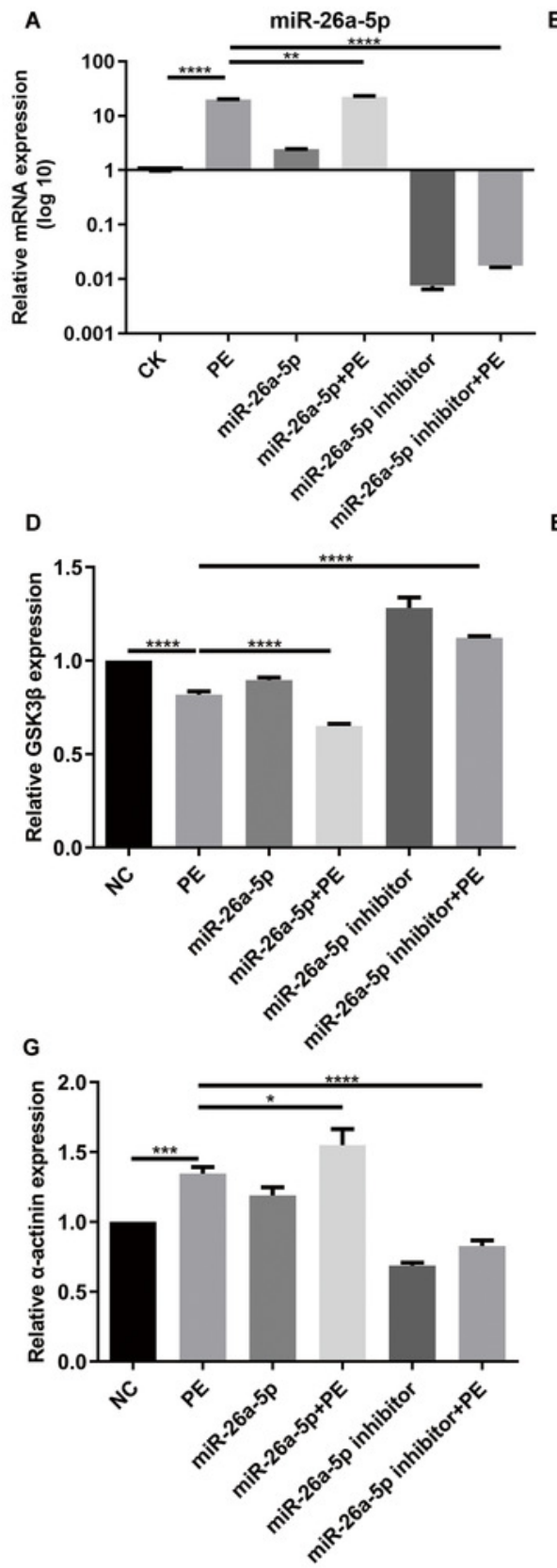

B

$$
\text { E }
$$

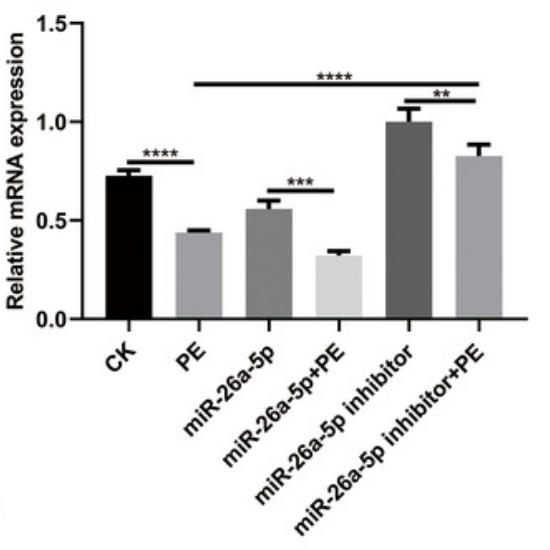

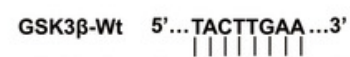

miR-26a-5p 3'...AUGAACUU...5'

GSK3ß-Mut $\quad 5^{\prime}, \ldots$ ATGAACTT ....
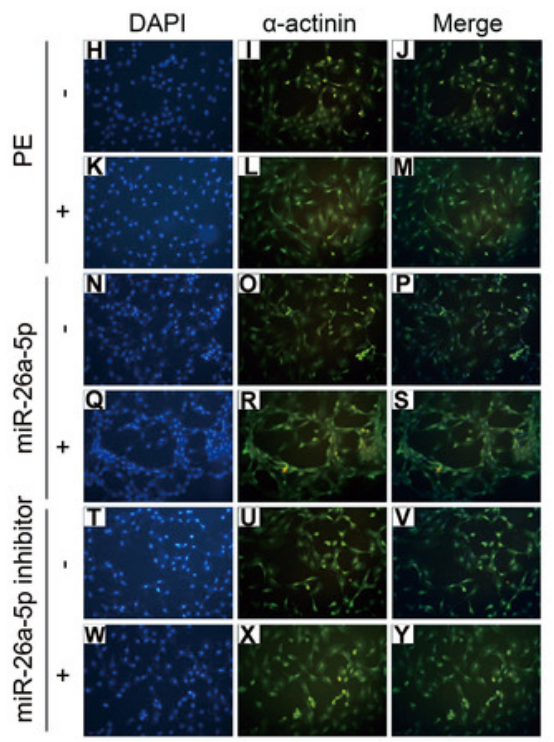

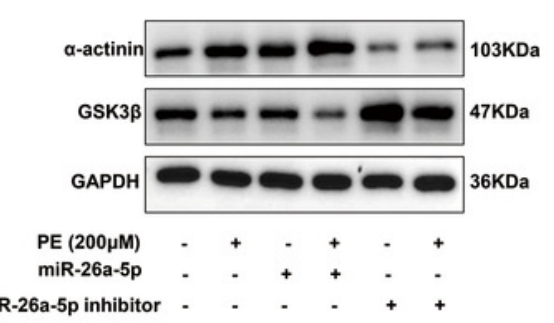

$\mathbf{F}$
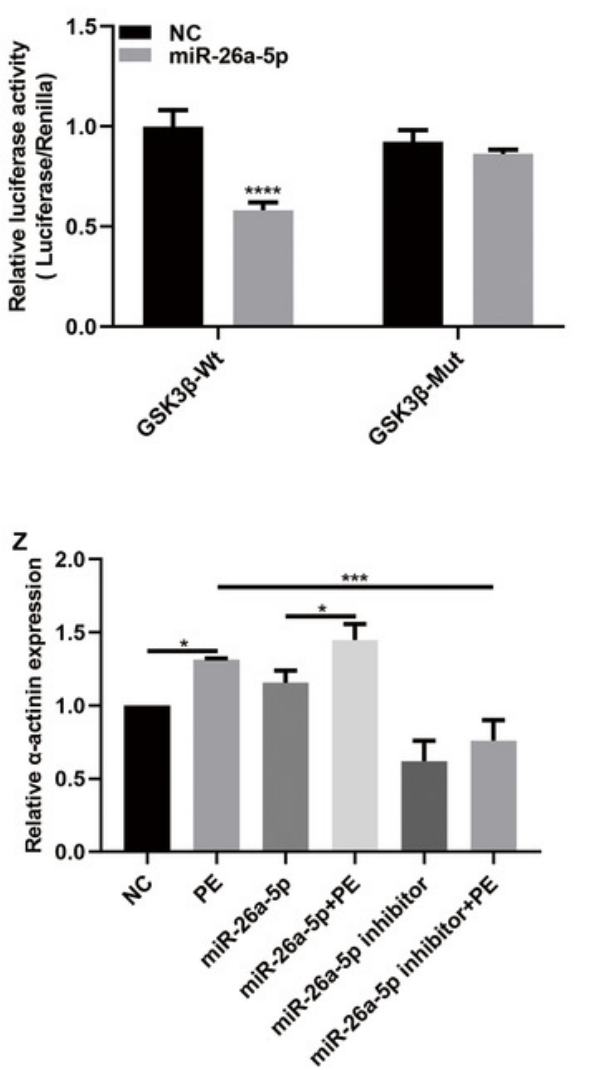
Figure 4

Effects of miR-26a-5p on the surface areas in PE-induced cardiac hypertrophy.

(A-R) Representative images of TRITC-phalloidin fluorescence staining. (S) Relative cardiomyocyte surface areas. Data were presented as mean \pm SD $(n=3) .{ }^{*} p<0.05 ;{ }^{* *} p<0.01$; $* * * * p<0.0001$.
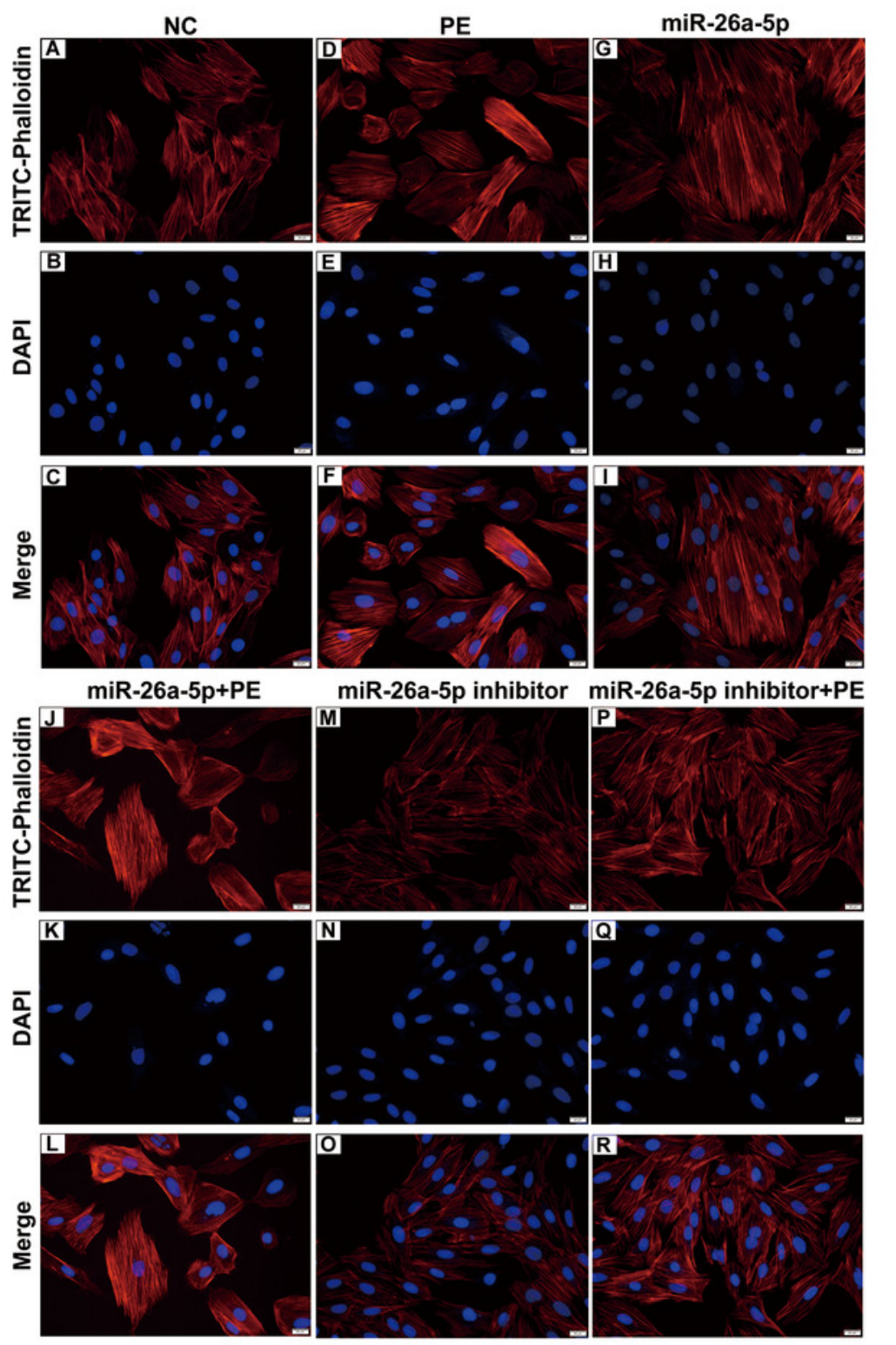

miR-26a-5p inhibitor miR-26a-5p inhibitor+PE
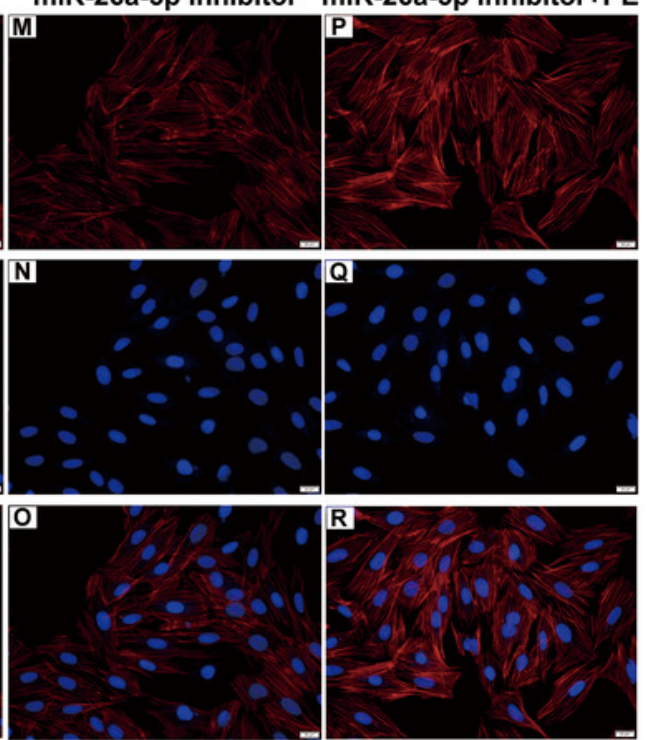

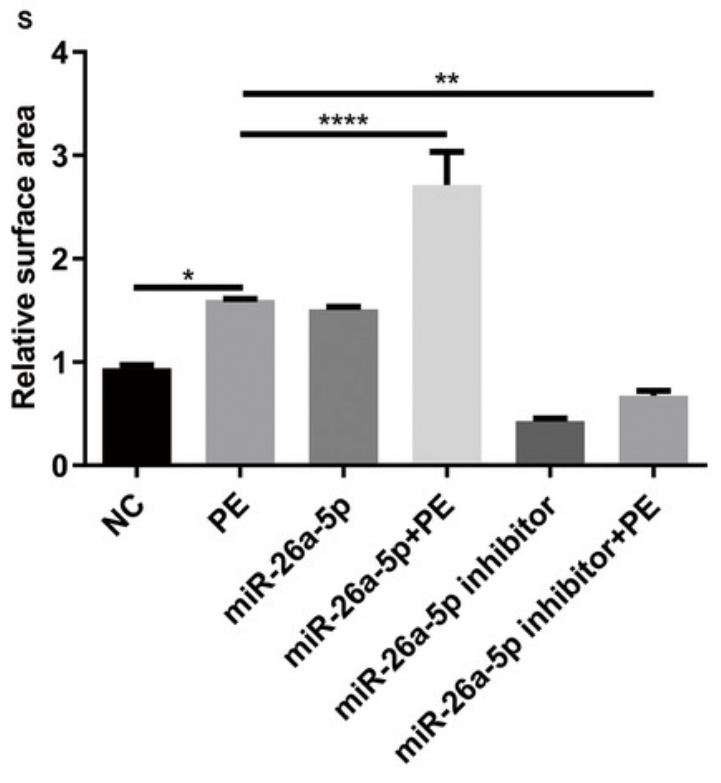




\section{Figure 5}

miR-26a-5p promotes cardiac hypertrophy by regulating GSK3 3 .

(A-C) qRT-PCR results showing the effects of miR-26a-5p on PE-induced H9C2 cells. (D, E) GSK3 $\beta$ was successfully overexpressed and silenced in H9C2 cells. (F-H) According to qRT-

PCR results, miR-26a-5p could promote the expression levels of cardiac hypertrophy-related markers by regulating GSK3 $\beta$. Data were presented as mean $\pm S D(n=3) .{ }^{* *} p<0.01$; $* * * p<0.001 ; * * * * p<0.0001$. 

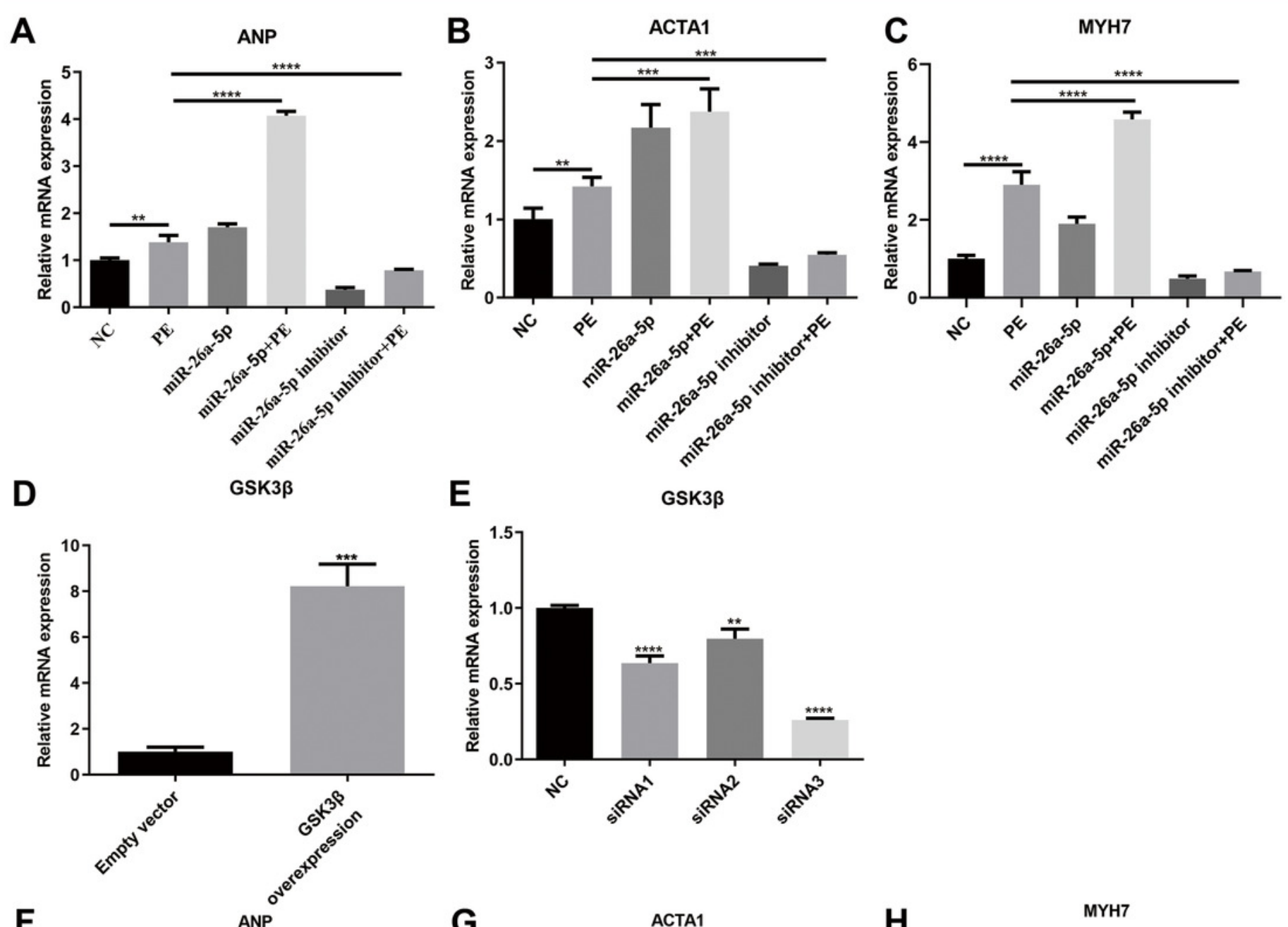

E

GSK3 $\beta$
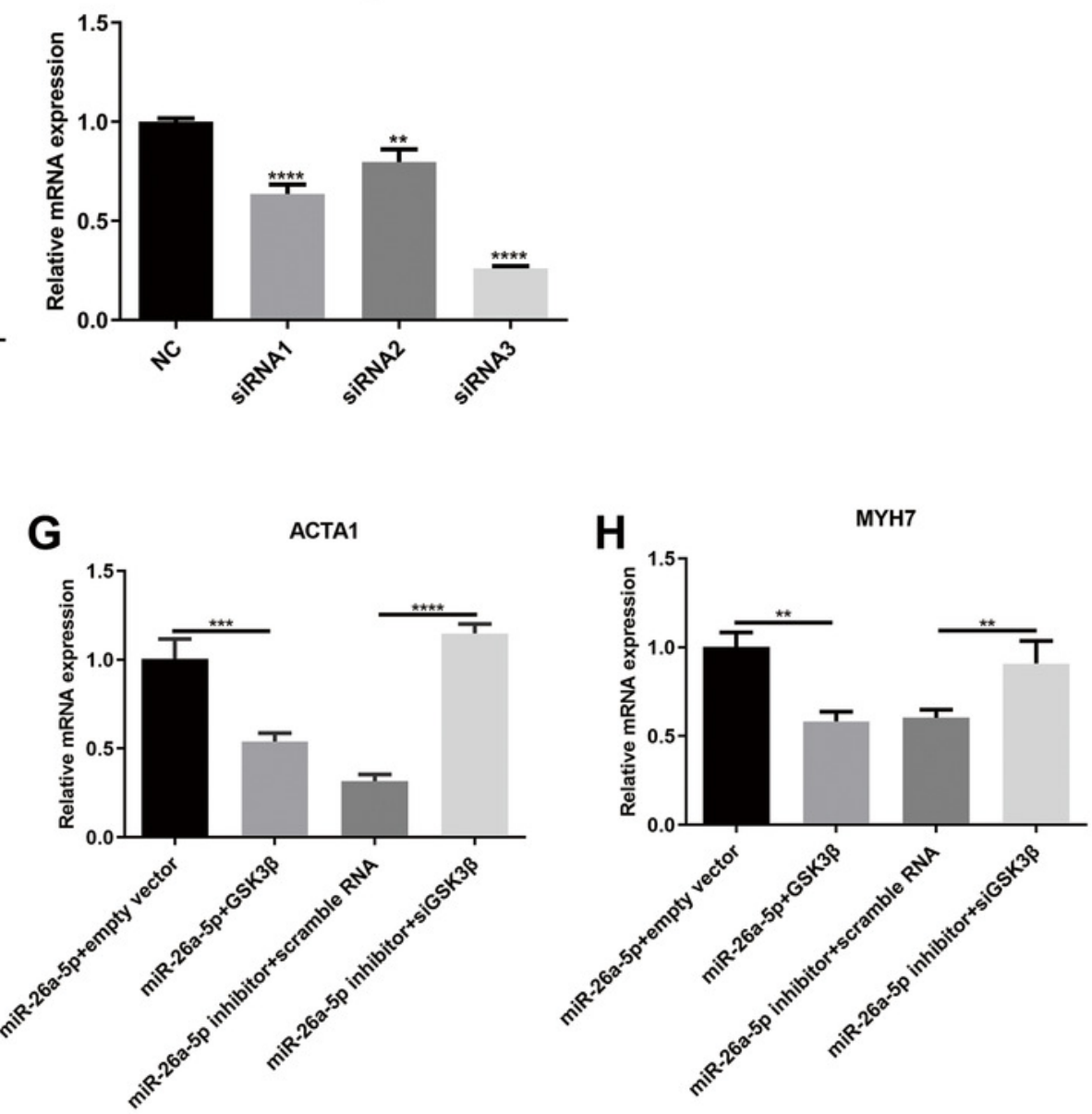


\section{Figure 6}

MiR-26a-5p inhibits GSK3 $\beta$ expression and promotes cardiac hypertrophy in vivo.

(A, B) qRT-PCR showing the mRNA expression levels of miR-26a-5p and GSK3 $\beta$ in SHR injected with miR-26a agomir. (C-G) The protein expression levels of GSK3 $\beta$, LC3II, Beclin-1 and p62 were detected in myocardial tissues by western blot. (H-P) Hematoxylin and eosin (H\&E) staining of myocardial tissues in the three groups $(\times 200)$. (Q-S) qRT-PCR results showing the mRNA expression levels of cardiac hypertrophy-related markers including ANP, ACTA1 and MYH7 in myocardial tissues. Data were presented as mean $\pm S D(n=3)$. **p-value $<0.01 ; * * * *$ p-value $<0.0001$; ns: no statistical significance. 

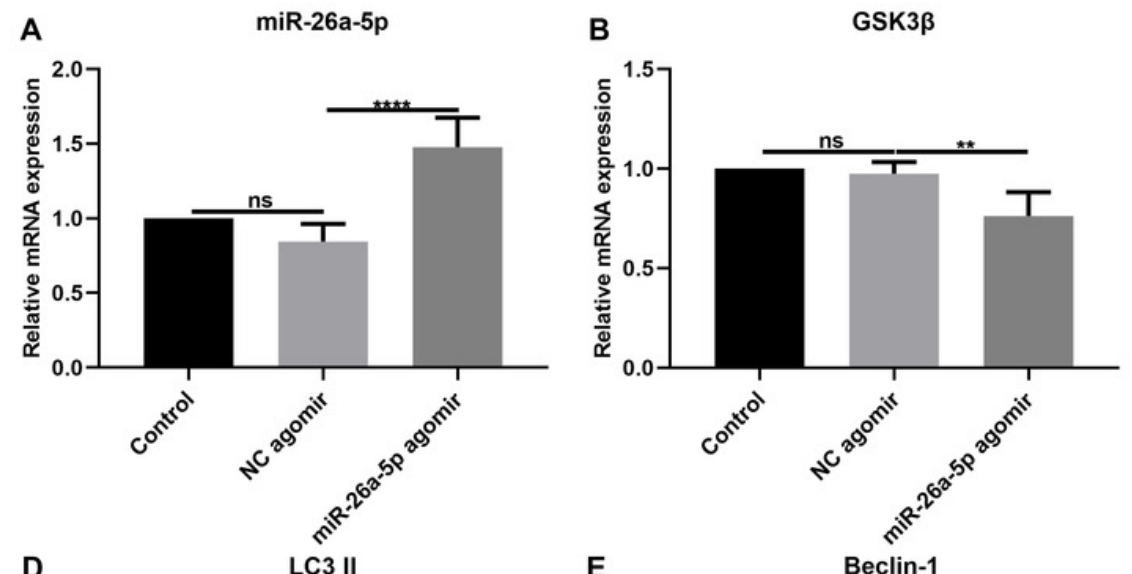

C GSK3 $\beta$

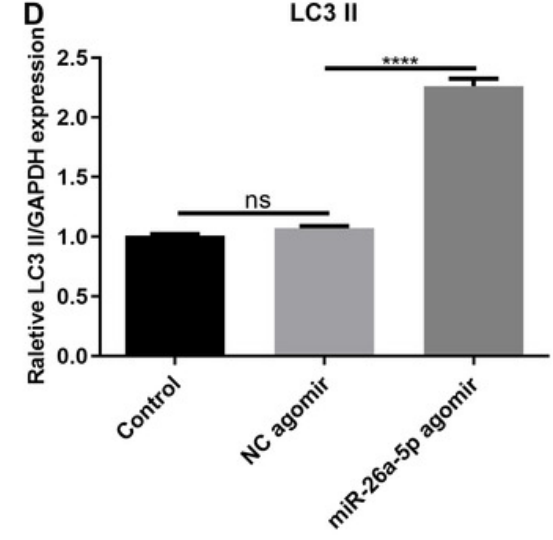

E
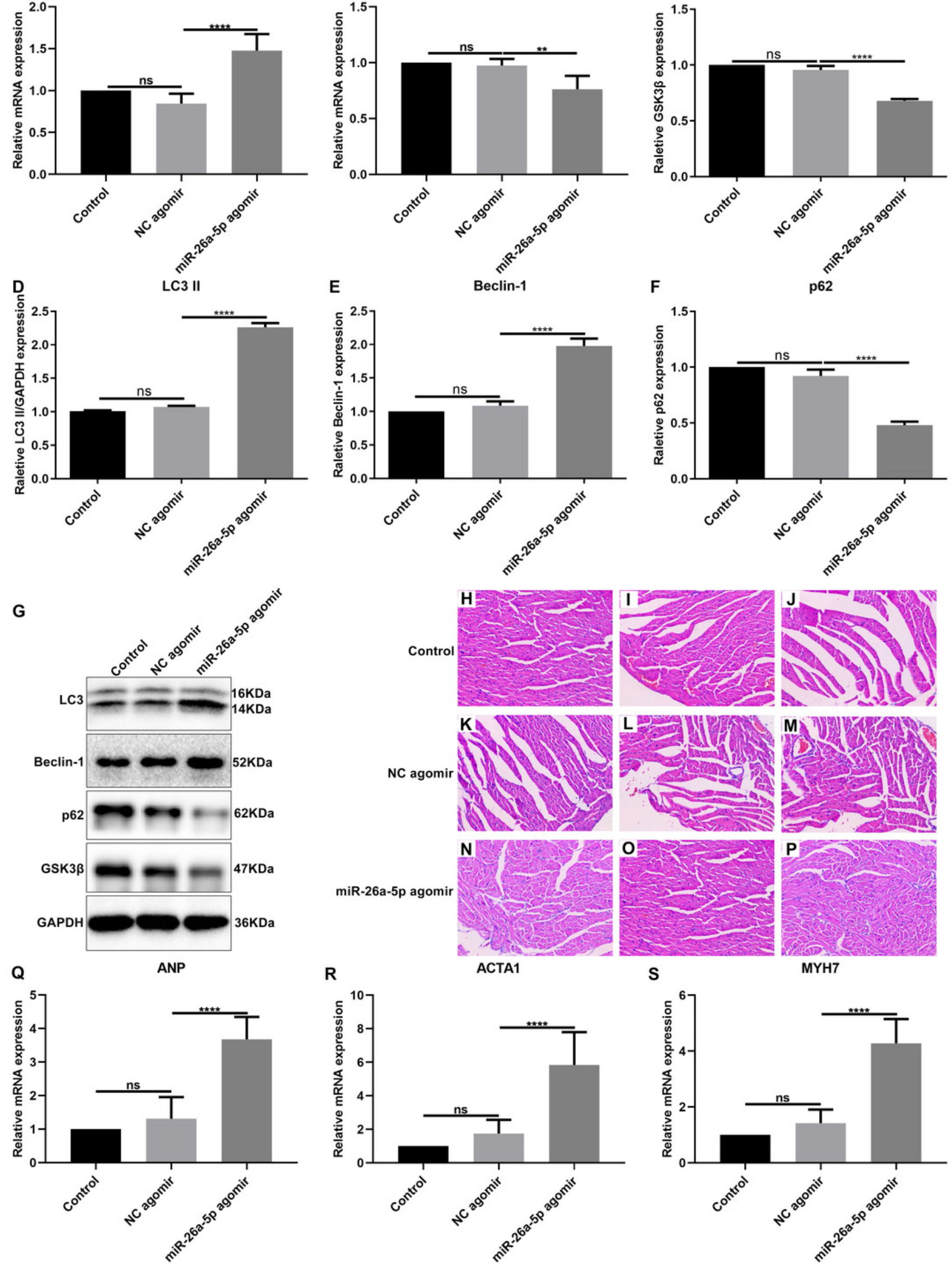